\title{
An analysis of Bordeaux meridian transit circle observations of planets and satellites (1997-2007)
}

\author{
J. E. $\operatorname{Arlot}^{1}$, G. Dourneau ${ }^{2}$, and J. F. Le Campion ${ }^{2}$ \\ ${ }^{1}$ Institut de Mécanique Céleste et de Calcul des Ephémérides, 77 avenue Denfert Rochereau, 75014 Paris, UMR 8028 du CNRS, \\ USTL, UPMC, France \\ e-mail: arlot@imcce.fr \\ 2 Université Bordeaux 1, Observatoire Aquitain des Sciences de l'Univers, Laboratoire d'Astronomie de Bordeaux, \\ UMR 5804 du CNRS, 2 avenue de l'Observatoire, 33270 Floirac, France \\ e-mail: dourneau@obs.u-bordeaux1.fr
}

Received 29 November 2007 / Accepted 12 February 2008

\section{ABSTRACT}

\begin{abstract}
Context. Meridian transit circle observations of the planets and their satellites are regularly performed for ephemerides improvement. Some were performed at the Bordeaux observatory during the period 1997-2007.

Aims. This paper presents these observations and analyses the data to determine the accuracy of the observations, their interest for dynamical purposes and compares them with the dynamical models of the observed objects.

Methods. For the determination of the positions of the planets, the observations of their satellites have been used, providing pseudo observations of the planets. The method is tested.

Results. The results show the interest of continuing this type of observation. Residuals show what ephemerides have to be improved using the present data.
\end{abstract}

Key words. astrometry - ephemerides - planets and satellites: general

\section{Introduction}

Bordeaux observatory continues to perform meridian transit circle observations and has included in its program of observations some solar system object. Since it appears that more solar system objects observations are needed and that the observations performed with other transit circle instruments are very useful, we tested the usefulness of our observations. The analysis of the reduction and of the comparison of observational data with ephemerides and with other observations made during the same period of time is given.

\section{The observations}

\subsection{The instrument}

The Bordeaux meridian transit circle is a $20 \mathrm{~cm}$ diameter refractor with a $2.37 \mathrm{~m}$ focal length. The derived scale of the instrument is $87 \mathrm{arcsec} / \mathrm{mm}$. The Bordeaux observatory is located at the following geodetic coordinates: Longitude $=$ $0 \mathrm{deg} 31 \mathrm{~min} 39 \mathrm{~s} \mathrm{~W}$, Latitude $=44 \mathrm{deg} 50 \mathrm{~min} 7 \mathrm{~s} \mathrm{~N}$ and Elevation $=73 \mathrm{~m}$. The Bordeaux meridian circle was fully automated from 1984 (Requieme \& Mazurier 1991). It was equipped with a photometric micrometer until 1994 when it was changed to a CCD camera $(512 \times 512$ pixels $)$ for tests and from 1996 the definitive camera $(1024 \times 1024)$ was used.

A two-stage thermoelectric Peltier unit is used to cool the Thomson $7896 \mathrm{M}$ CCD detector below $-40{ }^{\circ} \mathrm{C}$ in order to limit the resulting dark noise to about $65 \mathrm{e}-/ \mathrm{s}$. The size of the pixels is $19 \mu \mathrm{m} \times 19 \mu \mathrm{m}$, corresponding to $1.65 \operatorname{arcsec} \times 1.65 \operatorname{arcsec}$ in the sky. In declination, the field of the CCD chip is 28 arcmin. In right ascension, the transit time is $112 \mathrm{~s} / \cos \delta$ for stars with a declination of $\delta$. This time corresponds to the exposure time of the instrument when used in the drift scan mode.

Drift scan mode was used instead of classical stare mode. The scan mode is necessary in view of the characteristics of the transit observations and it allows us to observe a greater number of stars per night. Also, the rather long exposure time (about $112 \mathrm{~s}$ ) improves the limiting magnitude up to 16. As stars with a magnitude of 8.5 can be observed without significant pixel saturation, the dynamical range of the CCD unit appears to be about 7.5 mag. The strips observed in scan mode with the CCD detector are narrow in declination ( 28 arcmin) and can be much wider in right ascension, up to several hours. On the other hand, the scan mode presents some drawbacks, such as the distortion of star images which can reach a critical level at high declinations. Stars are rejected when their images present an elongation in right ascension six times higher than in declination. As a result, no observation can be obtained for declinations above 78 degrees. This is not a limitation for the observation of solar system objects orbiting near the equator.

Both GG495 and BG38 filters are used to select a reduced bandwidth of 5200-6800 $\AA$, with a central wavelength of $6050 \AA$, so as to limit the chromatic refraction to about 0.04 arcsec tanz. Today, the Bordeaux observatory meridian circle remains one of the last instruments of this type in regular operation, along with the FASTT in Flagstaff (Stone 1996), the CMASF in San Fernando, Argentina (Muinos et al. 2006) and the Valinhos meridian circle in Brazil (Viateau et al. 1999). For 
more detailed information about the Bordeaux meridian circle, see Viateau et al. (1996).

\subsection{The program of observations}

The regular observations of solar system objects such as Uranus and Neptune began in 1982 with a photoelectric micrometer (Rapaport et al. 1987). In 1996, with the automated CCD camera, we have continued this program observing new objects such as Pluto and the main satellites of Uranus, Ariel, Umbriel, Titania and Oberon, as well as Triton, the satellite of Neptune. Some of the major satellites of Saturn: Titan, Hyperion and Iapetus were observed from 1998. The planet Saturn was not observed, because of its too high brightness. Also, the image processing appeared not to be efficient enough to derive an accurate position of Saturn from its irregular shape, due to the vicinity of the rings. Some results of the first observations of Pluto and Saturn's satellites were obtained by Rapaport et al. (2002).

The Bordeaux meridian circle is currently involved in several other observing programs. The main program was Meridien 2000 , a systematic observation of the Bordeaux zone of the Astrographic Catalogue (11 deg $\leq \delta \leq 18 \mathrm{deg}$ ) over more than 3 years. A very consistent catalogue of positions and proper motions of all stars up to magnitude 15 has been derived (Rapaport et al. 2001; Ducourant et al. 2006; Rapaport et al. 2006). Other observing programs concern the asteroids, either to improve their masses (Viateau \& Rapaport 1996) or the accuracy of the prediction of star occultations from last minute observations. More recently, another program involving some extragalactic radiosources such as blazars, was developed in order to contribute to improve the ICRF system (Charlot \& Le Campion 2004).

\section{The reduction}

\subsection{Image processing}

The first step of the image processing is the extraction of the sky background. Two methods may be used. The first one consists of fitting the background to a polynomial of degree from 0 to 3 . This is a fast method but it can only be used for moderate gradients. In the case of planetary satellites, located in fields with stronger gradients, the median filter method is preferred. This method consists of searching the median value $\mathrm{M}$ of a square of $15 \times 15$ pixels centered on each pixel and subtract $M$ from the value of the central pixel.

After the extraction of sky background, the identification of objects is processed from the comparison of each pixel to the standard deviation $\sigma$ of the residuals of the pixels for each column. An object is identified when 2 consecutive pixels present residuals greater than $3 \sigma$. Two objects can be separated if their distance is greater than 5 arcsec. Then, the photo centre of the images is computed from a two-dimensional Gaussian fit. Bright objects with magnitudes lower than 8.5, such as some of the solar system objects involved in our observing program, present images with pixels that may be saturated. Such saturated pixels are rejected in the Gaussian fit, so as to limit consequent bias in the determination of the photo centre.

\subsection{Astrometric reduction}

For each star of each individual strip observed each night, the following system of equations is used to link the catalogued right ascension $\alpha_{\mathrm{R}}$, declination $\delta_{\mathrm{R}}$, and magnitude $V_{\mathrm{R}}$ of reference stars to their rectangular measured coordinates $(x, y)$ expressed in pixels and to the measured flux $\Phi$ in encoder step units as:

$$
\begin{aligned}
\alpha_{\mathrm{R}} & =\alpha_{0}+a_{1} x+a_{2}\left(y-y_{0}\right) \\
\delta_{\mathrm{R}} & =\delta_{0}+b_{1}\left(y-y_{0}\right)+b_{2} x+b_{3} \Phi \\
V_{\mathrm{R}} & =V_{0} 2.5 \log \Phi+c x
\end{aligned}
$$

$\alpha_{\mathrm{R}}$ and $\delta_{\mathrm{R}}$ are the catalogued positions of stars, reduced to the epoch of observation from their proper motions. $\alpha_{0}$ represents the local sidereal time at the instant of the beginning of the strip, and $\delta_{0}$ the declination of the centre of the strip $y_{0}$. The flux term in declination $b_{3} \Phi$ is used to recover the excessive shift of charge in the CCD for images of bright objects. As this effect appears to be negligible in right ascension, no flux term is used in this coordinate. The constants $a_{1}, a_{2}, b_{1}, b_{2}, b_{3}, V_{0}$ and $c$, are determined by the least-squares method, as well as $\alpha_{0}$ and $\delta_{0}$, also adjusted to observations. Then, the constants are used to determine the new positions and magnitude of the reference stars and of the other non-catalogued objects such as secondary stars, planets and satellites. A preliminary catalogue is obtained from the mean positions of all the stars common to the different strips, including secondary stars. The positions of this preliminary catalogue are reintroduced in the equations above for a second astrometric reduction. The convergence of the constants generally occurs after 5 successive iterations of this procedure. In order to limit atmospheric effects which can affect the observed positions, a curve is fitted on the residuals of each night of observations by the B-spline method (Viateau et al. 1999). The reference catalogue used is Tycho 2 providing data with an accuracy better than 60 mas for positions and 2.5 mas/year for proper motions (Hog et al. 2000). So, the positions of solar system objects observed by the Bordeaux meridian circle have a mean accuracy of about 60 mas. They are topocentric and given in the ICRF system and no chromatic correction has been made.

We made several attempts to evaluate the chromatic effects. The first was derived from previous work (Rapaport et al. 2001) made in the range of declination between 11 and 18 degrees of the M2000 catalog. Figure 1 shows the derived chromatic departure versus $(B-V)$ index. For this range of declination, Fig. 1 shows that chromatic departure is insignificant, as always under 20 mas, for objects with $(B-V)$ index between 0.5 and 1 , as most of solar system objects. In recent years, Saturn's satellites were located at a declination close to the M2000 zone. So, their positions presented in this paper must be insignificantly affected by chromatic effect and do not need any correction. However, Uranus, Neptune and Pluto were located at a negative declination from -10 degrees to -15 degrees during the recent period of observations. As the chromatic effect increases with the zenithal distance, the positions of these objects and their satellites can be significantly affected. Due to their low $(B-V)$ index, we have shown, by extrapolating the curve given in Fig. 1 to lower declinations, that Uranus and Neptune remain affected with very slight chromatic effects under 20 mas. Only their satellites Titania, Oberon and Triton, as well as the dwarf planet Pluto, due to their higher $(B-V)$ index, can be affected with a significant higher chromatic effect which nevertheless remains lower than positional errors. A second evaluation of chromatic effect involving many more stars, including low declination ones, has shown that this effect should be lower than 10 mas, whatever the declination and the $(B-V)$ index of the solar system objects. This last evaluation, obtained from real observations, appears to be more realistic than the first one which was derived from an extrapolation of observational effects. Finally, 
Table 1. Main characteristics of the observed objects.

\begin{tabular}{cccccccc}
\hline \hline Object & $\begin{array}{c}\text { mag } \\
\text { at } \\
\text { opp. }\end{array}$ & radius & radius & $\begin{array}{c}\text { phase } \\
\text { angle } \\
\text { arcsec } \\
\text { degrees }\end{array}$ & $\begin{array}{c}\text { phase } \\
\text { defect } \\
\text { mas }\end{array}$ & $\begin{array}{c}\text { orbital } \\
\text { period } \\
\text { days }\end{array}$ & $\begin{array}{c}\text { max } \\
\text { elong } \\
\text { arcsec }\end{array}$ \\
\hline Titan & 8.3 & 2440 & 0.35 & 6 & 13.8 & 15.9 & 209 \\
Hyperion & 14.2 & 200 & 0.03 & 6 & 1.3 & 21.3 & 254 \\
Iapetus & 11.9 & 720 & 0.10 & 6 & 3.5 & 79.3 & 610 \\
\hline Uranus & 5.5 & 24500 & 1.75 & 3 & 34.4 & - & - \\
Ariel & 14.4 & 580 & 0.04 & 3 & 8.1 & 2.5 & 15 \\
Umbriel & 15.3 & 585 & 0.04 & 3 & 8.1 & 4.1 & 21 \\
Titania & 13.9 & 800 & 0.06 & 3 & 12.3 & 8.7 & 35 \\
Oberon & 14.2 & 760 & 0.05 & 3 & 11.8 & 13.5 & 47 \\
\hline Neptune & 7.8 & 25100 & 1.15 & 2 & 15.0 & - & - \\
Triton & 13.7 & 1350 & 0.06 & 2 & 0.8 & 5.9 & 17 \\
\hline Pluto & 15.0 & 1200 & 0.05 & 2 & 0.7 & - & - \\
\hline
\end{tabular}

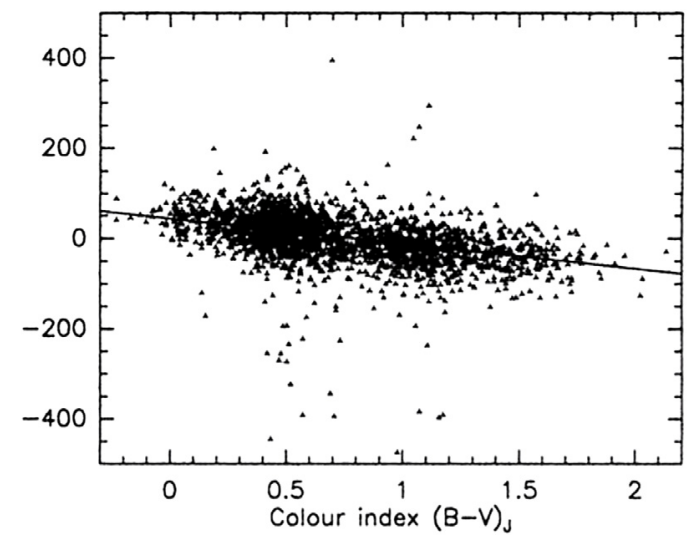

Fig. 1. Chromatic departure in declination vs. $(B-V)$ index, expressed in mas ( $\delta$ between 11 and 18 degrees).

no chromatic correction was made. This point still remains under consideration for the future.

\section{The data obtained}

The observations were carried out through the program of observations of the Bordeaux transit circle. Planets and their satellites were included in the program when possible. We did not include all the objects for several reasons. For some planets, it was unnecessary to make such observations because of the large sets of modern data (radar or from space probes) available, sufficient for dynamics studies (Mercury, Venus, Mars). For some planets such as Jupiter and Saturn, and some satellites, such as the Galilean satellites, the magnitude of the object did not allow observation. Also, the accuracy of the transit circle observations of Jupiter and Saturn will not be sufficient for dynamics studies. We were not able to observe the Galilean satellites, but we observed the Saturnian satellites. The good results obtained will allow us to solve the technical problems in order to observe the Galilean satellites. We will see that the positions of the satellites may be used to propose pseudo-observed positions of their primary that is very useful for Saturn: we observe a satellite, then we calculate the theoretical positions of the center of mass of the system and obtain a pseudo-position of the planet. The error on such a position is that of the dynamical model of the satellite which is much smaller than the error on the measurement of the position of the center of mass of the planet itself. In Table 1 we provide the main characteristics of the observed objects, in Table 2, the number of observations made, and in Table 3 the rms of each series of data calculated as follows:

$\mathrm{rms}=\sqrt{\frac{\Sigma(r-r m)^{2}}{(N-1)}}$

where $r$ is the $\mathrm{O}-\mathrm{C}$ on one observation and $r m$ the mean $(\mathrm{O}-\mathrm{C})$ for the series. $N$ is the number of observations. The ephemerides used are DE405 for the planets, TASS for the Saturnian satellites (Vienne \& Duriez 1995; Duriez \& Vienne 1997), LA06 by Lainey \& Arlot (2007) for the Uranian satellites and Jacobson (1991) for Triton. It is clear that the quality of the observations depends on the magnitude of the object, as shown in Fig. 2. For magnitudes greater than 14, the accuracy decreases rapidly as obvious in Table 3 for Hyperion and the Uranian satellites. Note that differences appear in right ascension and declination. These differences did not come from chromatic effects but confirm only that a meridian transit circle is more accurate in right ascension than in declination.

All the observational data have been published extensively in the Note Scientifique et Technique de l'IMCCE $\mathrm{n}^{\circ}$ S089 (Dourneau et al. 2007). They are available at the web address: http://www . imcce.fr/page.php?nav=fr/ publications/nst

\section{Comparison of observations with the ephemerides}

We calculated the $(\mathrm{O}-\mathrm{C})$ between the observations and the ephemerides in order to test the usefulness of the observations for the improvement of the dynamics of the observed objects. Figures 4 and 6 show the $(\mathrm{O}-\mathrm{C})$ in right ascension and declination as a function of time and Figs. 5 and 7 the $(\mathrm{O}-\mathrm{C})$ of all the observations. Note that the ephemerides are DE405 for the planets, TASS or Do93 by Dourneau (1993) for the Saturnian satellites and LJ86 (Laskar and Jacobson 1987) or LA06 for the Uranian satellites (Arlot et al. 2007).

Table 4 gives the values of the mean $(\mathrm{O}-\mathrm{C})$ s for each object and for each opposition. The motions of the planets are slow enough to have a very small variation of the $(\mathrm{O}-\mathrm{C})$ over one opposition (a few months), which is not true for the satellites which have a fast motion and thus, a variation of the $(\mathrm{O}-\mathrm{C}) \mathrm{s}$ with time over the same period. However, the mean $(\mathrm{O}-\mathrm{C})$ for the satellite over one opposition should be very close to the mean $(\mathrm{O}-\mathrm{C})$ for 

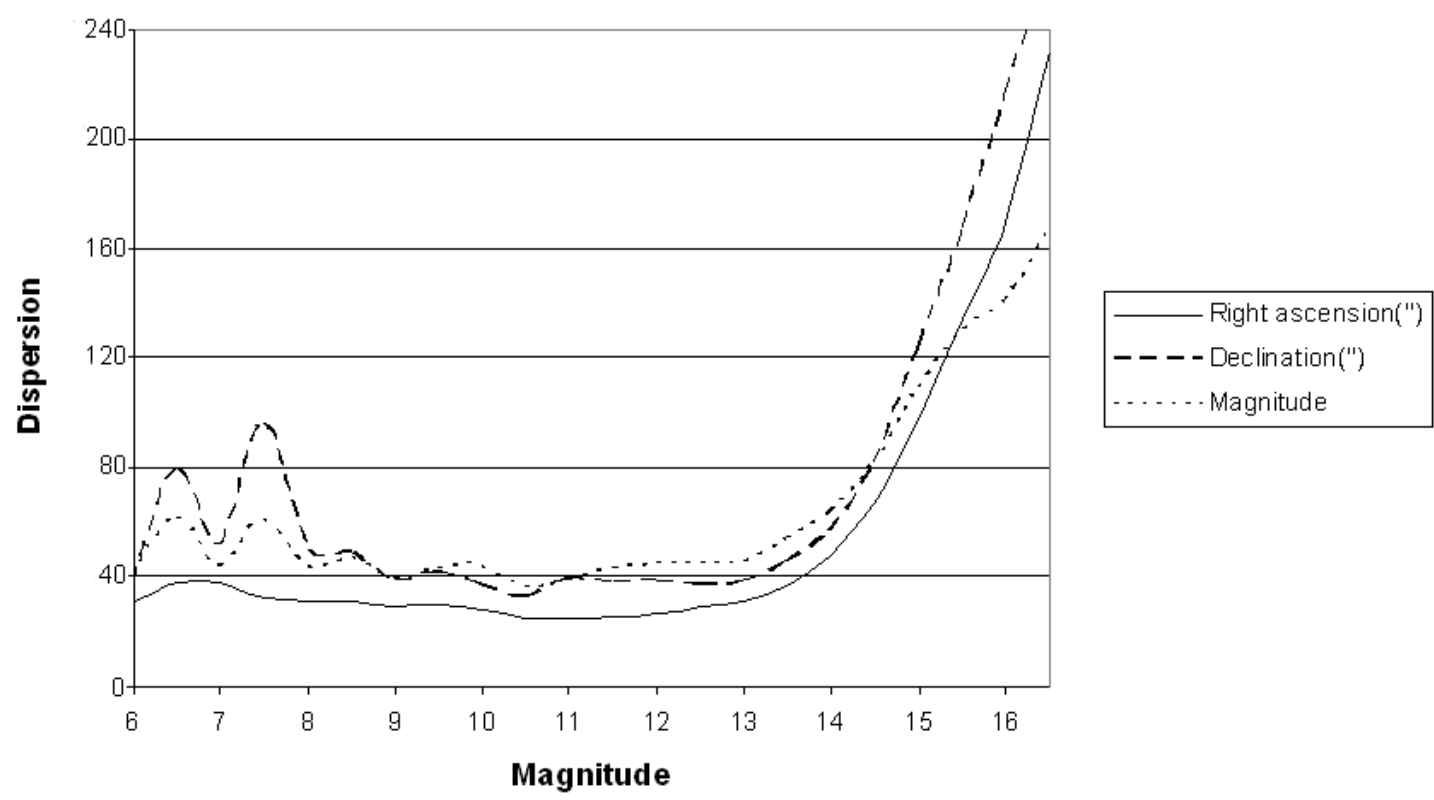

Fig. 2. Dispersion of the residuals (in mas) as a function of the $V$-magnitude.

Table 2. Number of observations.

\begin{tabular}{ccccccccccccc}
\hline \hline Object & 1997 & 1998 & 1999 & 2000 & 2001 & 2002 & 2003 & $2004-05$ & 2006 & 2007 & all \\
\hline Titan & - & - & 14 & - & 13 & - & 4 & 7 & 12 & 12 & 62 \\
Hyperion & - & - & 15 & - & 11 & - & 7 & 8 & 18 & 11 & 70 \\
Iapetus & - & - & 15 & - & 16 & - & 9 & 9 & 21 & 14 & 84 \\
\hline Uranus & 18 & 10 & 2 & - & 50 & 9 & 26 & 22 & 28 & - & 165 \\
Ariel & - & - & - & - & - & - & 2 & - & - & - & 2 \\
Umbriel & 2 & 2 & - & - & 1 & - & 6 & 2 & 7 & - & 20 \\
Titania & 14 & 5 & 1 & - & 29 & 5 & 14 & 12 & 16 & - & 96 \\
Oberon & 13 & 6 & 1 & - & 38 & 9 & 20 & 12 & 18 & - & 117 \\
\hline Neptune & - & - & 4 & - & - & - & 50 & 19 & 29 & - & 102 \\
Triton & - & - & 3 & - & - & - & 48 & 18 & 26 & - & 95 \\
\hline Pluto & - & - & - & - & - & 23 & - & - & 33 & - & 56 \\
\hline
\end{tabular}
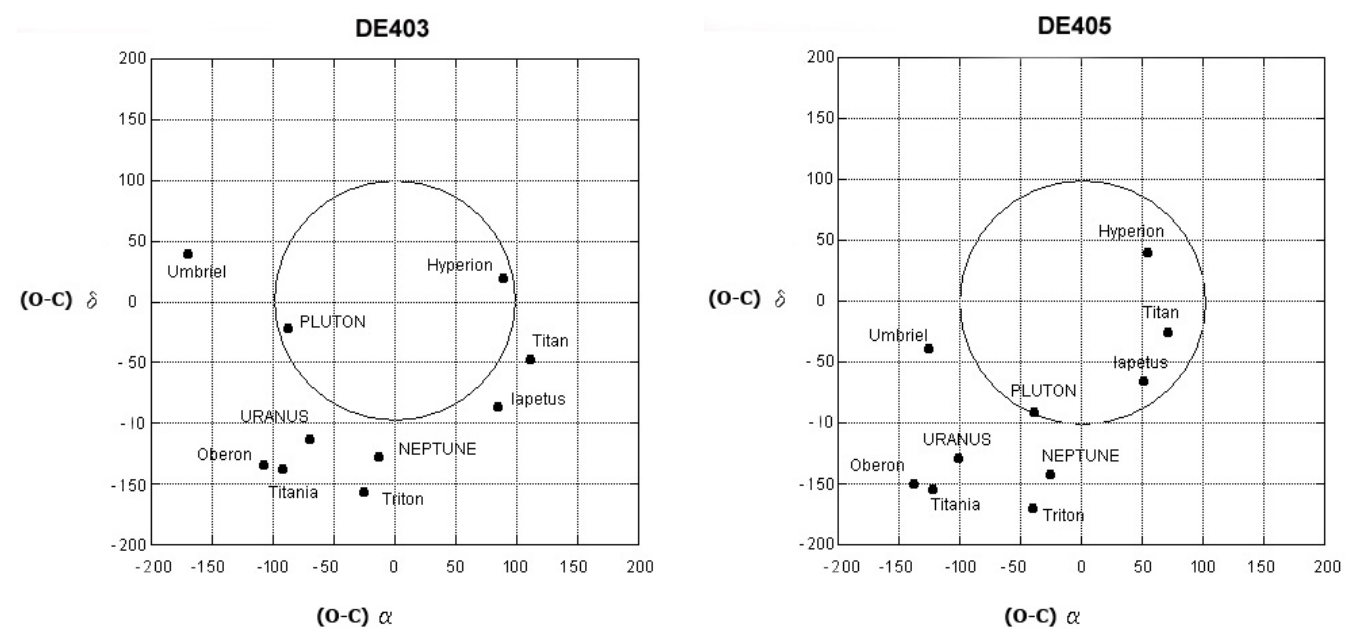

Fig. 3. Comparison of mean residuals $(\mathrm{O}-\mathrm{C})$ in mas derived from DE403 and from DE405.

the planet. For the Saturnian system, only three satellites were observed, showing similar $(\mathrm{O}-\mathrm{C}) \mathrm{s}$ which should correspond to the one of the planet. Hyperion, the magnitude of which is 14.2, has a poorer accuracy due to a low $\mathrm{S} / \mathrm{N}$ ratio. For the Uranian system, we find the same result. Contrarily to the Saturnian system, the planet itself has been observed and confirms that the satellites show similar $(\mathrm{O}-\mathrm{C})$ to the planet. Ariel should be excluded because of its poor observation history and Umbriel 

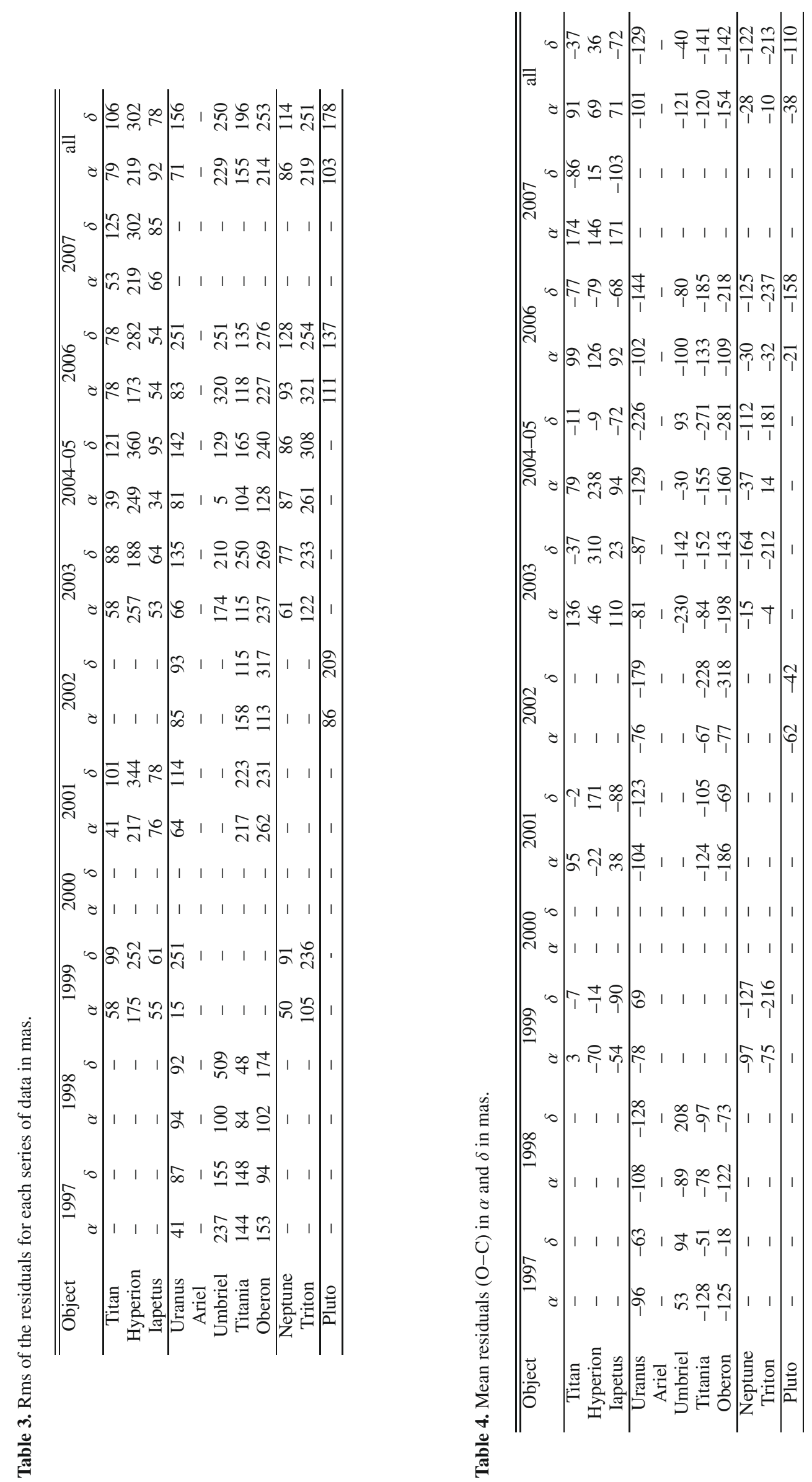

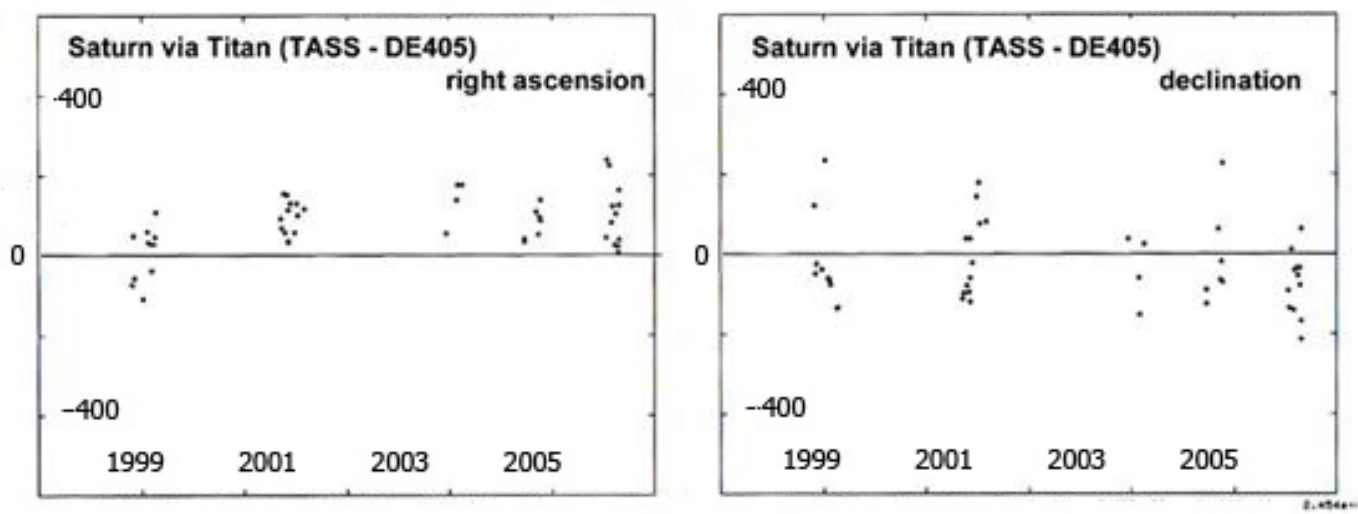

Fig. 4. Residuals $(\mathrm{O}-\mathrm{C})$ vs. time on the positions of Saturn deduced from the observed positions of Titan.
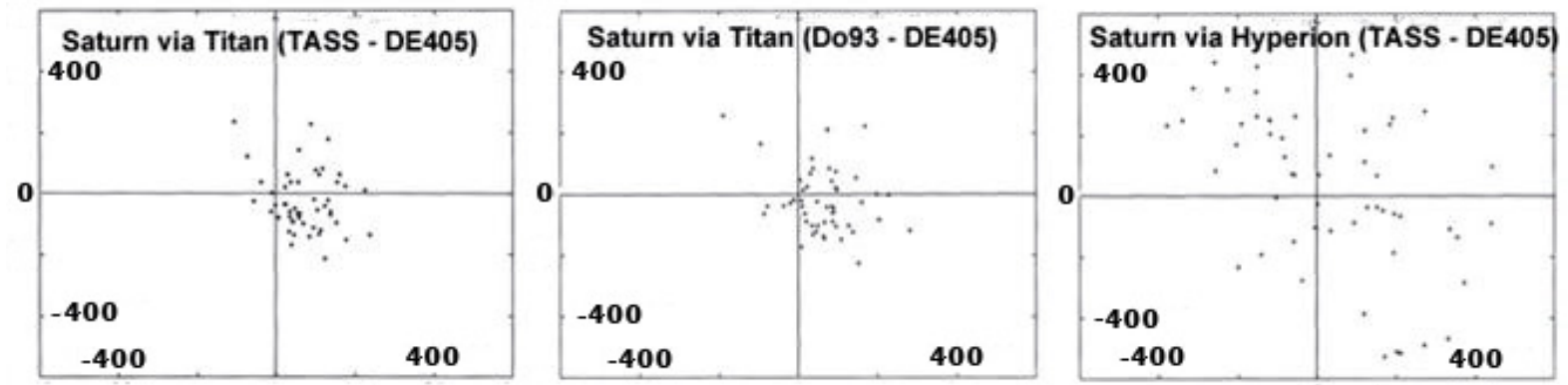

Fig. 5. Residuals $(\mathrm{O}-\mathrm{C})$ in $\alpha$ vs. $\delta$ on the positions of Saturn deduced from the observed positions of the satellites (a: from Titan TASS theory; b: from Titan Do93 theory; c: from Hyperion TASS theory).
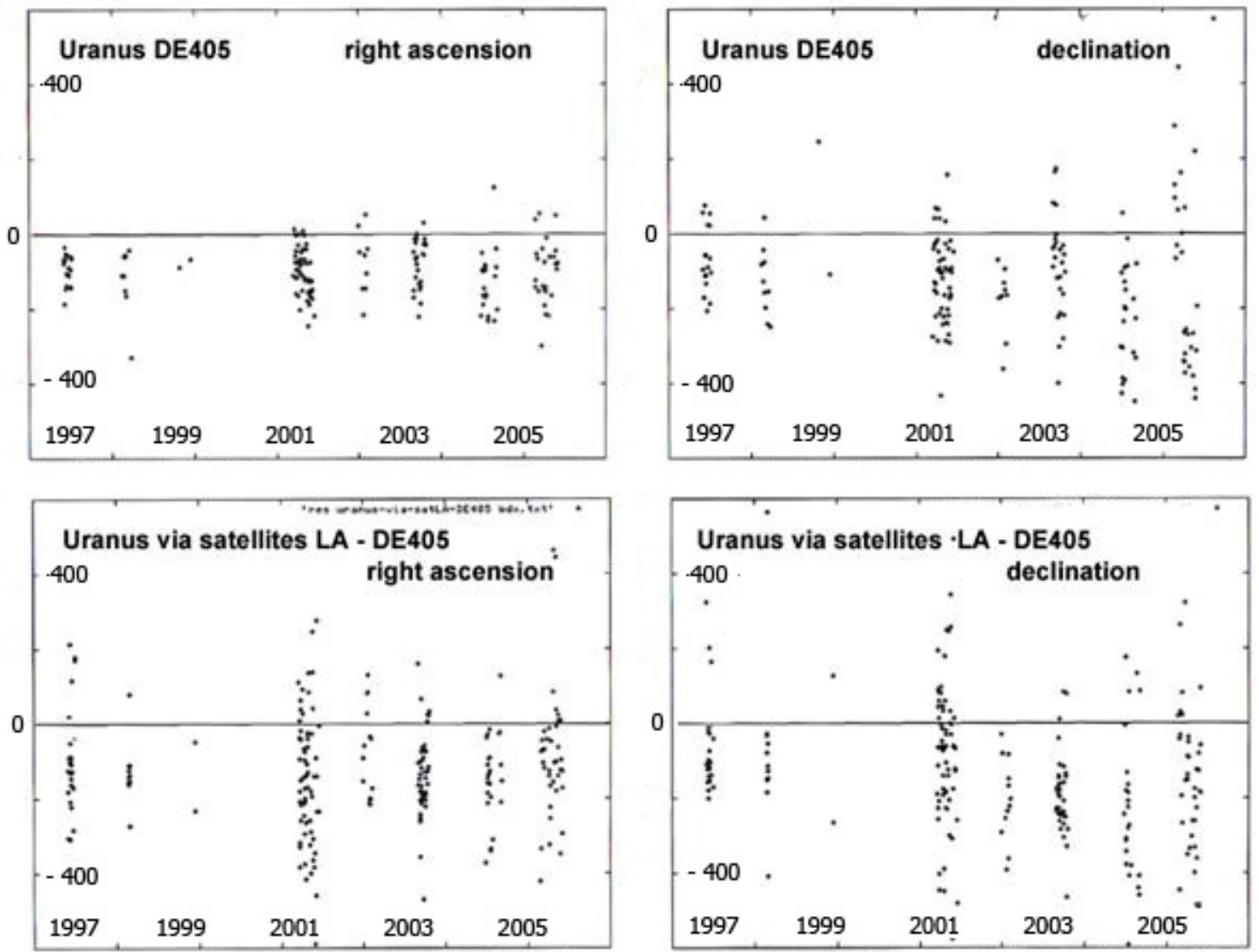

Fig. 6. Residuals $(\mathrm{O}-\mathrm{C})$ vs. time on the positions of Uranus directly observed and deduced from the observed positions of the satellites. 

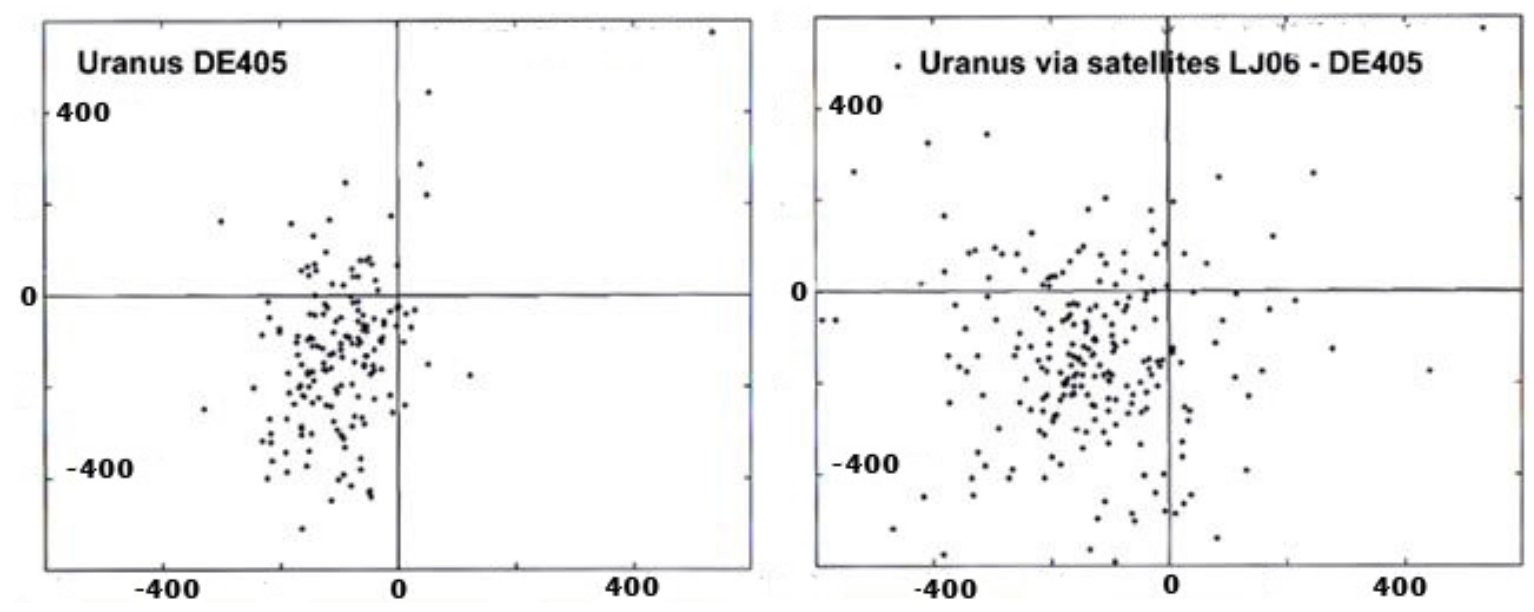

Fig. 7. Residuals (O-C) in $\alpha$ vs. $\delta$ on the positions of Uranus directly observed and deduced from the observed positions of the satellites.

Table 5. Mean residuals $(\mathrm{O}-\mathrm{C})$ in $\alpha$ and $\delta$ in mas for the period 1997-2005.

\begin{tabular}{ccccc}
\hline \hline Object & \multicolumn{2}{c}{ Bordeaux } & \multicolumn{2}{c}{ Flagstaff } \\
& $\alpha$ & $\delta$ & $\alpha$ & $\delta$ \\
\hline Uranus & -120 & -130 & -10 & -20 \\
\hline Umbriel & -120 & -40 & - & - \\
Titania & -120 & -140 & -100 & -80 \\
Oberon & -150 & -140 & -130 & -100 \\
\hline
\end{tabular}

Table 6. Mean residuals $(\mathrm{O}-\mathrm{C})$ in $\alpha$ and $\delta$ in mas for the 1998 opposition.

\begin{tabular}{ccccccc}
\hline \hline Object & \multicolumn{2}{c}{ Bordeaux } & \multicolumn{2}{c}{ Flagstaff } & \multicolumn{2}{c}{ Itajuba } \\
& $\alpha$ & $\delta$ & $\alpha$ & $\delta$ & $\alpha$ & $\delta$ \\
\hline Uranus & -110 & -130 & -160 & +40 & -330 & -10 \\
\hline Ariel & - & - & - & - & -140 & +40 \\
Umbriel & -90 & +210 & - & - & -160 & +50 \\
Titania & -80 & -100 & -140 & -20 & -130 & +90 \\
Oberon & -120 & -70 & -140 & -70 & -150 & +50 \\
\hline
\end{tabular}

because of poor astrometric results as consequence of its magnitude. For the Neptunian system, Triton's $(\mathrm{O}-\mathrm{C})$ s discrepancy is larger than that of Neptune and we do not find similar values. For Pluto, as well as for Uranus and Neptune, systematic negative $(\mathrm{O}-\mathrm{C}) \mathrm{s}$ appear.

In conclusion, assuming that the objects have a magnitude brighter than 15, the observations have an accuracy making them useful for dynamical purpose.

\section{Positions of planets derived from observed positions of satellites}

The accuracy of the observations of some bright satellites of the planets and the fact that their $(\mathrm{O}-\mathrm{C}) \mathrm{s}$ are mainly derived from the position of the planet, allows us to make pseudoobservations of the planets through the observation of their satellites. The dynamical models of the motion of the satellites may allow this, since the satellites are orbiting around the centre of mass of the system planet-satellites of interest. Note that the direct observation of the planet provides the position of an unknown point, requiring a model to determine the centre of mass. What theoretical model would be the best? Figure 4 shows the $(\mathrm{O}-\mathrm{C})$ of Saturn deduced from the observations of Titan, and
Table 7. Comparison of DE403 and DE405 ephemerides derived from mean residuals $(\mathrm{O}-\mathrm{C})$ in $\alpha$ and in $\delta$ of Bordeaux meridian circle 1997-2006 observations (in mas unit).

\begin{tabular}{ccccccc}
\hline \hline \multirow{2}{*}{ Object } & \multirow{2}{*}{ Period } & $N$ & \multicolumn{4}{c}{ DE403 residuals (O-C) } \\
& & & $\alpha$ & $\delta$ & $\alpha$ & $\delta$ \\
\hline Titan & $1999-2006$ & 50 & 111 & -47 & 71 & -26 \\
Hyperion & $1999-2006$ & 59 & 89 & 20 & 54 & 40 \\
Iapetus & $1999-2006$ & 70 & 84 & -86 & 51 & -66 \\
\hline Uranus & $1997-2005$ & 165 & -70 & -113 & -101 & -129 \\
Ariel & $1998-2003$ & 2 & -1775 & 561 & -1797 & 546 \\
Umbriel & $1997-2005$ & 20 & -88 & -22 & -125 & -39 \\
Titania & $1997-2005$ & 96 & -92 & -138 & -122 & -154 \\
Oberon & $1997-2005$ & 113 & -108 & -134 & -138 & -150 \\
\hline Neptune & $1999-2005$ & 102 & -13 & -128 & -26 & -142 \\
Triton & $1999-2005$ & 89 & -26 & -156 & -40 & -170 \\
\hline Pluto & $2002-2005$ & 54 & 170 & 40 & -39 & -91 \\
\hline
\end{tabular}

Fig. 5, the $(\mathrm{O}-\mathrm{C}) \mathrm{s}$ in right ascension versus declination for all the observations. The ephemerides DE405 of Saturn show a systematic offset. Figure 5a shows the $(\mathrm{O}-\mathrm{C}) \mathrm{s}$ when using the TASS ephemeris for Titan, Fig. 5b when using Do93 Dourneau ephemeris for Titan. The offset to DE405 is similar, showing the weak impact of the theoretical model of the motion of the satellite on the pseudo observations of the planet. Contrarily, the impact of the chosen satellite used for the pseudo observation of the planet is very large. Figure $5 \mathrm{c}$ shows what happens when using Hyperion instead of Titan. The discrepancy is very large and the observation of Saturn deduced from that of Hyperion is poorly accurate. This was obvious when looking at Table 3: the rms of the residuals reach 0.22/0.30 arcsec for Hyperion and 0.08/0.11 for Titan for all the series of data. In the case of Iapetus, the accuracy of the measurement is better than for Hyperion but the theoretical model is worse than the one of Titan which encourages us to use Titan to determine pseudo-positions of the planet.

\section{Comparison of the observations with other sets of data made at the same time - comparison of the (O-C)s from DE403 and DE405}

Since similar series of observations are made at other observatories, it is interesting to compare the results obtained. From 1997 to 2005, observations were performed at Flagstaff using FASTT 
Table 8. Mean residuals $(\mathrm{O}-\mathrm{C})$ in $\alpha$ and $\delta$, and standard errors on the residuals $\left(\sigma_{\alpha}, \sigma_{\delta}\right)$ in mas of the Saturnian satellites for three available theories.

\begin{tabular}{cccccccc}
\hline \hline Object & Period & $N$ & Theory & $(\mathrm{O}-\mathrm{C}) \alpha$ & $(\mathrm{O}-\mathrm{C}) \delta$ & $\sigma_{\alpha}$ & $\sigma_{\delta}$ \\
\hline Titan & $1999-2007$ & 62 & & & & & \\
& & & TASS & 91 & -37 & 120 & 111 \\
& & & Do93 & 87 & -36 & 126 & 113 \\
& & & HT93 & 89 & -35 & 133 & 116 \\
\hline Hyperion & $1999-2007$ & 70 & & & & & \\
& & & TASS & 69 & 36 & 228 & 302 \\
& & & Do93 & 523 & -114 & 876 & 365 \\
& & & HT93 & 111 & -51 & 752 & 356 \\
\hline Iapetus & $1999-2007$ & 84 & & & & & \\
& & & TASS & 71 & -72 & 115 & 106 \\
& & & Do93 & 32 & -81 & 263 & 195 \\
& & & HT93 & 8 & -89 & 311 & 133 \\
\hline
\end{tabular}

transit circle facilities. Since Uranus moves slowly, we can compare the $(\mathrm{O}-\mathrm{C}) \mathrm{s}$ derived from Flagstaff and Bordeaux. Table 5 provides the mean $(\mathrm{O}-\mathrm{C}) \mathrm{s}$ calculated using the LJ86 ephemeris for the satellites and DE 405 for the planet. For Titania and Oberon, both series of observations are in agreement, but for the planet Uranus, the results disagree. The observations from Bordeaux for Uranus are in agreement with the observations of the satellites, and that is not the case for Flagstaff. Table 6 provides a comparison of the $(\mathrm{O}-\mathrm{C})$ s obtained for the opposition of 1998, with also, observations made at Itajuba, Brazil, with a classical CCD target on a $1.6 \mathrm{~m}$-telescope (Veiga \& Vieira-Martins 1999). The results are much more in agreement except for Uranus itself, probably because of the brightness of the planet which decreases the observational accuracy.

Mean residuals in Table 7 are displayed in Fig. 3 with residuals in declination versus residuals in right ascension. Figure 3 shows that the DE405 ephemeris presents no real improvement on the DE403, excepted for Saturn. For Saturn, this result is in good agreement with Rapaport et al. (2002). For Uranus and Neptune, we confirm the very small discrepancies between both ephemerides, previously mentioned by Rapaport et al. (2002). Figure 3 shows that such discrepancies are less than 20 mas. They are favourable for DE403 but this is not significant as we are below the observational accuracy. For Pluto, Fig. 3 also confirms the improvement of DE405 in right ascension and the theoretical difference DE405-DE403 of about 100 mas in declination presented by Rapaport et al. (2002). But in this case, we can see in Fig. 3 that this significative difference in Pluto declination does not appear to be in favour of DE405. The most important improvement of DE405 with respect to DE403 is obtained for Saturn. This can be due to the fact that only new accurate observations of Saturn derived from spacecraft data were used in the DE405 in order to improve the DE403 ephemeris.

\section{Comparison of the theories of motion of Saturn's satellites.}

Table 8 shows that most of the lowest standard errors are derived from TASS theory by Vienne \& Duriez (1995) and by Duriez \& Vienne (1997). However, the lowest absolute mean residuals are generally obtained for the other theories Do93 (Dourneau 1987, 1993) for Titan in right ascension and HT93 (Harper \& Taylor 1993; Taylor 1992) for Titan in declination as well as for Iapetus in right ascension.
For Hyperion, TASS theory presents all the lowest mean and standard residuals. This means that TASS has improved HT93 Taylor's theory (1992) which had previously improved Do93 Dourneau's theory (1987). The latest theory appears to need, for this satellite, a major improvement so as to include a series of perturbation terms, in comparison to Taylor and TASS theories.

This analysis shows that TASS theory, presenting most of the lowest standard errors, proposes the best model representing the real orbits of Saturnian satellites. However, this theory appears to need an improvement so a new fit to observations can be made in order to reduce the significant shift that we have observed in right ascension and in declination.

TASS appears to give the best model of orbits as it considers additional terms not included in other theories. But both of the other theories Do93 and HT93 present a better fit to observations than TASS theory for Iapetus in right ascension and for Titan in right ascension and in declination.

\section{Conclusion}

Transit circle observations are still useful for observations of planetary satellites and for planets. The pseudo positions of the planets deduced from the observation of satellites are valuable, mainly for Jupiter and Saturn, the centers of mass of which are not easy to measure directly, and also for Uranus, increasing the amount of data since Uranus itself is measurable. These observations have an accuracy similar to that of the direct observations. These observations have allowed us to obtain some results concerning the consistency of planetary and satellite theoretical models. For the planets we have shown that the DE405 ephemeris presents no real improvement on the DE403 ephemeris, except for Saturn. For the satellites of Saturn, the TASS theory appeared to give the best model of their orbits, but we have shown that the other models Do93 and HT93 can present a better fit to the observations for some satellites such as Titan and Iapetus. We encourage the continuation of such regular automatic observations allowing a sample of observations well distributed over time.

Acknowledgements. This paper has been made possible thanks to the staff of the meridian circle of Bordeaux Observatory. 


\section{References}

Arlot, J. E., Lainey, V., \& Thuillot, W. 2007, A\&A, 456, 1173

Charlot, P., \& Le Campion, J. F. 2004, in Highlights 2004, ed. F. Combes, et al. (EDP Sciences), 425

Dourneau, G. 1987, These de Doctorat d'État, Université Bordeaux 1

Dourneau, G. 1993, A\&A, 267, 292

Ducourant, C., Le Campion, J. F., Rapaport, M., et al. 2006, A\&A, 448, 1235

Dourneau, G., Le Campion, J. F., Rapaport, M., et al. 2007, Note scientifique et technique de l'IMCCE n ${ }^{\circ} 089$, Paris

Duriez, L., \& Vienne, A. 1997, A\&A, 324, 366

Harper, D., \& Taylor, D. B. 1993, A\&A, 268, 326

Hog, E., Fabricius, C., Makarov, V. V., et al. 2000, A\&A, 357, 367

Jacobson, R. A., Reidel, J. E., \& Taylor, A. H. 1991, A\&A, 247, 565

Laskar, J., \& Jacobson, R. A. 1987, A\&A, 118, 212

Muinos, J. L., Belizon, F., Vallejo, M., et al. 2006, Rev. Mex. Astron. Astrofis., 25,47
Rapaport, M., Requieme, Y., Mazurier, J. M., \& Francou, G. 1987, A\&A, 179, 317

Rapaport, M., Le Campion, J.-F., Soubiran, C., et al. 2001, A\&A, 376, 325

Rapaport, M., Teixeira, R., Le Campion, J. F., et al. 2002, A\&A, 383, 1054

Rapaport, M., Ducourant, C., Le Campion, J. F., et al. 2006, A\&A, 449, 435

Requieme, Y., \& Mazurier, J. M. 1991, A\&AS, 89, 311

Stone, R. C. 2001, AJ, 122, 2723

Stone, R. C., \& Harris, F. H. 2000, AJ, 119, 1985

Stone, R. C., Ronald, C., Monet, D. G., et al. 1996, AJ, 111, 4

Taylor, D. B. 1992, A\&A, 265, 825

Veiga, C., \& Vieira-Martins, R. 1999, A\&AS, 138, 247

Viateau, B., \& Rapaport, M. 1996, A\&A, 334, 729

Viateau, B., Réquiéme, Y., Le Campion, J. F., et al. 1999, A\&AS, 134, 173

Vienne, A., \& Duriez, L. 1995, A\&A, 297, 508 